\title{
Cross-match as an immuno-oncological risk factor for hepatocellular carcinoma recurrence and inferior survival after living donor liver transplantation: A call for further investigation
}

\author{
Cheng-Maw HO*, Rey-Heng HU, Yao-Ming WU, Ming-Chih HO, Po-Huang LEE \\ Department of Surgery, National Taiwan University Hospital, Taipei, Taiwan
}

Introduction: The success of immunotherapy for patients with hepatocellular carcinoma (HCC) suggest that immune dysregulation occurs in HCC patients. This warrants an immuno-oncological risk assessment in the platform of liver transplantation.

Methods: This retrospective single-center study analyzed risk factors for-particularly cross-matching performed through conventional complement-dependent cytotoxicity cross-match tests-and the outcomes of HCC recurrence following living donor liver transplant.

Results: A total of 71 patients were included. The median follow-up period was 29.1 months. Seventeen $(23.9 \%)$ patients had posttransplant HCC recurrence, and their 1-, 3-, and 5-year survival rates were 70.6\%, 25.7\%, and 17.1\%, respectively, which were inferior to those of patients without HCC recurrence $(87.0 \%, 80.7 \%$, and $77.2 \%$, respectively $[p<0.001])$. In addition to microvascular invasion, positive cross-match results for $\mathrm{B}$ cells at $37^{\circ} \mathrm{C}\left(\mathrm{B}-37^{\circ} \mathrm{C}\right)$ or $\mathrm{T}$ cells at $4^{\circ} \mathrm{C}\left(\mathrm{T}-4^{\circ} \mathrm{C}\right)$ was associated with inferior overall survival in multivariable analysis after adjustment for tumor status beyond Milan criteria and elevated alpha-fetoprotein levels. Rejection alone cannot be the mechanism underlying the effects of positive cross-match results on patient outcomes. Adjusted survival curves suggested that positive cross-match $\mathrm{B}-37^{\circ} \mathrm{C}$ or $\mathrm{T}-4^{\circ} \mathrm{C}$ was associated with inferior recurrence-free and patient survival, but the robustness of the finding was limited by insufficient power.

Conclusions: Additional large-scale studies are required to validate positive cross-match as an immuno-oncological factor associated with HCC recurrence and inferior patient survival. 